\title{
5 Land und Materialität
}

„Land is not like a mat. You cannot roll it up and take it away. “1 Mit diesem Bild von Land als Matte, die nicht einfach zusammengerollt und an einen anderen Ort getragen werden kann, erinnert uns die kanadische Kulturanthropologin Tania Li an ein wesentliches Charaktermerkmal raumbezogener Neuaushandlungsprozesse um Land. Land, so die vermeintlich offensichtliche, aber dennoch häufig übersehene Erkenntnis Lis, ist materiell und physisch greifbar. Die Materialität und örtliche Fixiertheit von Land bedeutet, wie oben ausgeführt, dass Land im Gegensatz zu anderen natürlichen Ressourcen weder „abgebaut“, noch „verpflanzt“ oder an anderer Stelle „verwertet“ werden kann. Die mit großflächigen Landinvestitionen einhergehenden Kommodifizierungs-, Assetisierungs- und Inwertsetzungsprozesse sind somit eng an die zeit-räumlichen Grenzen agrarischer Produktion gebunden.

Trotz dieser so augenscheinlich zentralen Eigenschaft von Land wurde die Bedeutung seiner Materialität für die Raumpraxis großflächiger Agrarinvestitionen bislang selten in den Blick genommen. Die Literatur zum globalen Land Rush problematisierte vor allem die mit dem Erwerb von Agrarflächen einhergehenden Verteilungs- und Nutzungspolitiken und stellte damit Dimensionen wie Territorium, Besitz und Eigentum in den Vordergrund. Materielle und biophysische Aspekte von Land - über die natürliche Beschaffenheit des Bodens, den Einfluss des Klimas, bis hin zu agrarischen Praktiken wie der Verwendung von oder dem Verzicht auf Chemikalien - blieben in der Analyse meist außen vor. ${ }^{2}$ In diesem Abschnitt diskutieren wir die Rolle, die die spezifische Materialität von Land im Kontext aktueller Landtransformationen spielt. Wir skizzieren zunächst die besondere Gebundenheit von Land und Landwirtschaft an materielle und biologische Prozesse und geben im Anschluss drei Beispiele für die Verflechtung von Land und Materialität im Kontext der Finanzialisierung von Land.

$1 \mathrm{Li}$, ,What is Land?“.

2 D. Münster und J. Poerting, „Land als Ressource, Boden und Landschaft. Materialität, Relationalität und neue Agrarfragen in der Politischen Ökologie“, Geographica Helvetica 71 (2016), S. 245-257. Auch das kürzlich erschienene Sonderheft der Fachzeitschrift Agriculture and Human Values betrachtet die Bedeutung der Materialität von Land und Boden im Kontext von Landinvestitionen, siehe Sippel und Visser, „Reimagining Land“. 


\subsection{Land und Landwirtschaft als biologische Systeme}

Die Vernachlässigung der Rolle materieller und biophysischer Aspekte für Erscheinungsformen und Auswirkungen der globalen Landnahme ist bemerkenswert, da die Produktivität von Land in einem Umfang, wie dies vielleicht bei keiner anderen natürlichen Ressource der Fall ist, an Ort und Zeit gebunden ist. Während landwirtschaftliche Produkte im Zuge der fortschreitenden Globalisierung von Handels- und Warenketten zunehmend aus ortsgebundenen Interaktionszusammenhängen herausgelöst werden ${ }^{3}$ - und die Konsumtion von agrarischen Gütern damit zeit-räumlich entgrenzt wird -, bleibt die landwirtschaftliche Produktion selbst an saisonale und biophysische Wachstums- und Arbeitszyklen gebunden. Jahreszeitliche Schwankungen, Extremwetterereignisse oder das Auftreten von Schädlingen setzen der landwirtschaftlichen Intensivierung und Output-Generierung natürliche Grenzen und stellen aus Sicht von Investoren ein nicht unerhebliches Investitionsrisiko dar. ${ }^{4}$ Die materielle Ortsgebundenheit von Land und Landwirtschaft steht hierbei in einem zunehmenden Spannungsverhältnis mit den auf landwirtschaftlicher Produktion beruhenden Waren- und Finanzflüssen innerhalb des globalen Ernährungssystems. Gertel und Sippel sprechen in diesem Zusammenhang von einer „Ungleichheit“ raum-zeitlicher Logiken innerhalb landwirtschaftlicher Austauschprozesse: Dem materiellen Austausch landwirtschaftlicher Güter über weite räumliche Distanzen hinweg sowie der virtuellen, oftmals sekundenschnellen Transaktion von Finanzflüssen an den Agrar-Terminmärkten der Welt steht ein landwirtschaftlicher Produktionsprozess gegenüber, der weiterhin von Saisonalität und lokalen Wachstumszyklen geprägt ist. $^{5}$

Die Erkenntnis, dass sich die der Landwirtschaft innewohnende „Logik des Lebenden“6 immer wieder den Akkumulationsanforderungen kapitalistischer

3 C. Reiher und S. R. Sippel, „Einleitung. Umkämpftes Essen in globalen Kontexten“, in: C. Reiher und S. R. Sippel (Hrsg.), Umkämpftes Essen: Produktion, Handel und Konsum von Lebensmitteln in globalen Kontexten, Göttingen: Vandenhoeck \& Ruprecht, 2015, S. 9-37, hier S. 18.

4 B. Kuns, O. Visser und A. Westfält, „The Stock Market and the Steppe. The Challenges Faced by Stock-Market Financed, Nordic Farming Ventures in Russia and Ukraine“, Journal of Rural Studies 45 (2016), S. $199-217$.

5 J. Gertel und S. R. Sippel, „The Financialisation of Agriculture and Food“, in: M. Shucksmith und D. L. Brown (Hrsg.), Routledge International Handbook of Rural Studies, New York: Routledge, 2016, S. 215-226.

6 F. Jacob, La Logique du Vivant. Une Histoire de l'Hérédité, Paris: Gallimard, 1974, zitiert in J. Auderset und P. Moser, Die Agrarfrage in der Industriegesellschaft. Wissenskulturen, Machverhältnisse und natürliche Ressourcen in der agrarisch-industriellen Wissensgesellschaft (18501950), Köln: Böhlau, 2018, S. 28. 
Wachstumsgesellschaften entzieht, ist nichts Neues. Bereits seit dem Aufkommen der sogenannten „Agrarfrage“ in der zweiten Hälfte des 19. Jahrhunderts wurde deutlich, dass sich die Landwirtschaft aufgrund ihrer Naturabhängigkeit der vollständigen Standardisierung, Technisierung, Monetarisierung und Industrialisierung - kurz, den Produktionsparadigmen des modernen Industriekapitalismus - nicht bruchlos und allumfassend unterordnen lässt. ${ }^{7}$ Agrarsoziolog:innen sprachen daher von einem „Exzeptionalismus“ der Landwirtschaft, ${ }^{8}$ da sich diese nicht nahtlos in die Verwertungsprozesse moderner Wachstumsgesellschaften einpassen lasse. Noch in den späten 1990er Jahren ging man davon aus, dass trotz der zunehmenden Macht von Konzernen innerhalb des Ernährungssystems die landwirtschaftliche Primärproduktion selbst wenig attraktiv für Industrie- und Finanzinteressen sei und somit auf absehbare Zeit in den Händen von Familienund Kleinbetrieben verbleiben würde - freilich bei zunehmender Unterwerfung solcher Betriebe unter die Zwänge kapitalistischer Wachstumslogiken in Form der Ausweitung vertragslandwirtschaftlicher Beziehungen zwischen bäuerlichen Betrieben und der von Großkonzernen geprägten Verarbeitungsindustrie. ${ }^{9}$

Umso erstaunlicher erscheint vor diesem Hintergrund das neu erwachte Interesse kaufkräftiger Akteure aus Industrie und Finanzwirtschaft an der Agrarproduktion. Das Argument, dass es den Akteuren dieses gegenwärtigen Land Rushs vor allem um den lukrativen Wiederverkauf des Landes zu gestiegenen Bodenpreisen - also eher um eine Form der „virtuellen“ als „materiellen“ Akkumulationslogik - ginge, greift hier zu kurz. Kapitalwertsteigerungen ländlicher Flächen sind, wenn man von Fällen der Spekulation auf Landnutzungsänderung (der Umwandlung von günstigerem Agrarland in teureres Bauland) absieht, stets auch an materielle Faktoren wie die Produktivität und den Output der jeweiligen Agrarflächen gebunden. Dies bedeutet im Umkehrschluss, dass auch Investoren mit einem eher kurzfristigen Anlagehorizont, wie Private Equity Fonds, ein nicht unerhebliches Maß an Arbeit und Kreativität in die Organisation und Kontrolle von materiellen Produktionsprozessen auf den von ihnen erworbenen Agrarflächen investieren, wie Ouma am Beispiel Tansanias aufzeigt. ${ }^{10}$ Aus dem histori-

7 Ebd., S. 27-28.

8 S. Mann und J. Dickinson, „Obstacles to the Development of a Capitalist Agriculture“, Journal of Peasant Studies 5 (1987) 4, S. 466 - 481.

9 R. C. Lewontin, „The Maturing of Capitalist Agriculture. Farmer as Proletarian“, in: F. Magdoff, J. B. Foster und F. H. Buttel (Hrsg.), Hungry for Profit. The Agribusiness Threat to Farmers, Food, and the Environment, New York: Monthly Review Press, 2000, S. 93-106. Siehe auch W. D. Heffernan, „Concentration of Ownership and Control in Agriculture“, in: ebd., S. 61-75.

10 S. Ouma, Farming as Financial Asset. Global Finance and the Making of Institutional Landscapes, Newcastle upon Tyne: Agenda Publishing, 2020. 
schen Blickwinkel der Agrarfrage betrachtet ist der gegenwärtige Ansturm finanzkräftiger Investoren auf Agrarland damit nicht nur empirisch, sondern auch theoretisch ein bemerkenswertes Phänomen.

Dass sich Investoren trotz der historischen „Widerständigkeit“ der Landwirtschaft gegenüber den zeit-räumlichen Verwertungslogiken des modernen Kapitalismus derzeit verstärkt auf den Erwerb von Agrarland konzentrieren, ist jedoch - wie oben erwähnt - paradoxerweise auch den materiellen und biophysischen Eigenschaften von Land selbst geschuldet. Als greifbare, beständige und in Zeit und Raum eindeutig zu verortende Ressource erscheint Land im Vergleich zu den oftmals hochgradig intransparenten und „virtuellen“ Anlageprodukten internationaler Finanzmärkte von eher geringer Komplexität. Land galt als sichere Anlageklasse - nicht trotz, sondern gerade aufgrund seiner Materialität. Doch auch für die finanzökonomischen Inwertsetzungsprozesse, die sich im Kontext des neuen Ansturms auf Land beobachten lassen, spielen materielle und biophysische Eigenschaften von Land eine entscheidende Rolle. Drei Formen dieser in der Materialität von Land verankerten Wertzuschreibungen sollen im Folgenden detaillierter aufgezeigt werden.

\subsection{Verknappungsdiskurse}

Eine Kerndimension der Inwertsetzung von Agrarland im Kontext des gegenwärtigen Land Rushs ist die Kultivierung eines Alarmszenarios: das Schwinden ländlicher Flächen und damit einhergehende Engpässe in der Versorgung der Weltbevölkerung mit Nahrungsmitteln. Innerhalb der Investorendiskurse wurden globales Bevölkerungswachstum, eine steigende Nachfrage nach Anbauflächen für Agrarbrennstoffe im Kontext von Peak Oil und Klimawandel sowie sich wandelnde Ernährungsgewohnheiten in asiatischen Schwellenländern als Haupttriebkräfte dieser prognostizierten Verknappung von Agrarland identifiziert. Steil ansteigende Preise für Lebensmittel wie zuletzt während der globalen Nahrungsmittelpreiskrisen von 2007/08 und 2011 sowie die gesteigerte Investitionstätigkeit „neuer“ Akteure aus dem Nahen Osten (zum Beispiel Katar) und Asien (zum Beispiel China) im globalen Ernährungssystem seien Belege, so das gängige Narrativ, für die zunehmende Knappheit von Land und eine global steigende Nachfrage nach Nahrungsmitteln. Die Behauptung einer existenzbedrohenden Verknappung von Land geht einher mit gesteigerten Renditeerwartungen. Als materiell begrenzte Ressource lassen Investitionen in Land und die Primärproduktion von Nahrungsmitteln in diesem Szenario nicht nur auf stabile, sondern gar auf wachsende Einkommensströme hoffen - eine Inwertsetzungslogik, die in internationalen Investorenzirkeln häufig mit dem Mark Twain zugeschrie- 
benen Ausspruch „buy land, they're not making it anymore“ zum Ausdruck gebracht wird. ${ }^{11}$

Dass Ressourcenknappheit kein rein „natürliches“ Phänomen, sondern auch sozial produziert und von Machtungleichheiten durchdrungen ist, wird hierbei von involvierten Finanzakteuren wie auch politisch Verantwortlichen genauso übersehen wie die Tatsache, dass Verknappungsdiskurse nicht immer den tatsächlichen Gegebenheiten vor Ort entsprechen. So zeigten Kuns u. a. am Beispiel der Investitionen skandinavischer Agrarkonzerne in Russland und der Ukraine, dass die Nachfrage nach Agrarland in diesen Regionen deutlich hinter den Erwartungen des von Investorenkreisen konstruierten Alarmszenarios zurückblieb - mit entsprechend negativen Auswirkungen, so zumindest aus Sicht der beteiligten Finanzakteure, auf Bodenpreissteigerungen und Renditeerwartungen. $^{12}$

\subsection{Bodenfruchtbarkeit und Produktivität}

Neben der tatsächlichen oder diskursiv erzeugten Knappheit von Land und den sich daraus ergebenden Erwartungen an Bodenpreissteigerungen ist das Rekurrieren auf grundlegende materielle Eigenschaften von Land eine weitere wichtige Dimension bei der Inwertsetzung von Land als Investitionsobjekt. ${ }^{13} \mathrm{Zu}$ diesen materiellen Eigenschaften zählen neben Bodenbeschaffenheit und Fruchtbarkeit auch geomorphologische Aspekte einer Ackerfläche - so sind zum Beispiel Flachlandflächen aufgrund ihrer Möglichkeit zur maschinellen Bewirtschaftung Äckern in bergigen Regionen vorzuziehen. Auch geographische Lage, landwirtschaftliche Infrastruktur und Bewässerungsmöglichkeiten lassen Rückschlüsse auf die zu erwartende Produktivität von Land zu. Bei der Bestimmung und Bewertung der materiellen Eigenschaften von Land spielt die „material agency and potentiality“ 14 von Land eine wichtige Rolle, ebenso wie Prozesse der Standardisierung und Normierung, durch die Bodeneigenschaften über zeit-räumliche Bezüge hinweg erfasst und vergleichbar gemacht werden.

Die Schwarzerde-Regionen in der Ukraine sowie in Teilen Russlands sind ein prominentes Beispiel dafür, wie biophysische und materielle Eigenschaften bei

11 O. Visser, „Running out of Farmland? Investment Discourses, Unstable Land Values and the Sluggishness of Asset Making“, Agriculture and Human Values 34 (2017), S. 185-198, hier S. 191. 12 Kuns, Visser und Westfält, „The Stock Market and the Steppe“.

13 Visser, „Running out of Farmland?“.

14 T. Richardson und G. Weszkalnys, „Resource Materialities“, Anthropological Quarterly 87 (2014) 1, S. 5-30, hier S. 15. 
der Konstruktion von Land als Anlageobjekt diskursiv mobilisiert werden. Schwarzerde-Böden, auch Tschernosem genannt, gelten seit Jahrhunderten als einer der fruchtbarsten Bodentypen weltweit und idealer Ackerboden. Visser zeigt, dass die Schwarzerde-Regionen Mitteleuropas seit Anfang der 2000er Jahre auch die Aufmerksamkeit internationaler Finanzakteure erregt haben. ${ }^{15}$ Das Interesse internationaler Investoren an Schwarzerde-Böden liegt maßgeblich in der ihnen zugeschriebenen hohen Fruchtbarkeit und Produktivität begründet. Angepriesen als „phänomenal produktives Land“ mit „unglaublichem Potential“, ${ }^{16}$ wurde Schwarzerde-Böden diskursiv eine Werthaftigkeit zugeschrieben, die einen regelrechten Ansturm kapitalkräftiger Akteure - von börsennotierten Agrarkonzernen und chinesischen Staatsunternehmen bis hin zu internationalen Finanzinvestoren - auf diese Landflächen ausgelöst hat. Umso erstaunlicher erscheint, dass viele der Investitionsprojekte weit hinter ihren selbstgesteckten Renditeerwartungen zurückblieben. Visser argumentiert, dass die eindimensionale Fokussierung auf die Bodenqualität andere, für die landwirtschaftliche Produktion zentrale, aber weniger „greifbare“ Aspekte wie die rauen klimatischen Bedingungen, Dürreanfälligkeit und unregelmäßige Regenfälle in den Hintergrund treten ließen. Ernteausfälle und schwere wirtschaftliche Verluste waren die Folge. ${ }^{17}$

Das Beispiel der Schwarzerde-Investitionen zeigt, wie eng Diskurs und Materialität im Ansturm auf Land miteinander verwoben sind. Während spezifische materielle Aspekte von Land und Landwirtschaft ihre Reimagination als neue Finanzanlageklasse befeuern, setzen ihre materiellen und biophysischen Eigenheiten den Investorenträumen von einer nahtlosen kapitalgetriebenen Verwertbarkeit von Land immer wieder Grenzen. ${ }^{18}$ Eben jene Prozesse der Simplifizierung und Abstrahierung, durch welche Land für den kapitalistischen Verwertungsprozess „lesbar“ gemacht werden, können die erfolgreiche Konstruktion von Land als Investitionsobjekt unterminieren, indem sie den Blick auf nur einen Aspekt wie den der Bodenqualität - innerhalb eines hochkomplexen biologischen Prozesses verengen.

15 O. Visser, N. Mamonova und M. Spoor, „Oligarchs, Megafarms and Land Reserves. Understanding Land Grabbing in Russia“, The Journal of Peasant Studies 39 (2012) 3-4, S. 899-931. 16 Visser, „Running out of Farmland?“, S. 195.

17 O. Visser, „Persistent Farmland Imaginaries. Celebration of Fertile Soil and the Recurrent Ignorance of Climate“, Agriculture and Human Values 38 (2021) 1, S. 313-326.

18 Sippel und Visser, „Reimagining Land“. 


\subsection{Vermarktung von Umwelt und Natur}

Stehen bei der Inwertsetzung von Schwarzerde-Böden noch „objektive“ (wenn auch einseitig gedachte) Faktoren landwirtschaftlicher Produktivität im Vordergrund, so lassen sich im Kontext des Land Rush auch Formen der kulturellen und symbolischen Wertzuschreibung erkennen, bei denen es Investoren um die Vermarktung von Umwelt und Natur im weiteren Sinne geht. So zeigt Böhme am Beispiel der Investitionspraxis chinesischer Konzerne in Australien, wie Zuschreibungen von „Ursprünglichkeit“ und „Natürlichkeit“ zu wichtigen Vermarktungsinstrumenten gegenüber chinesischen Konsumenten werden. Vor dem Hintergrund von Umweltverschmutzung und Lebensmittelskandalen in China positionieren chinesische Investoren ihre in Australien produzierten Milchprodukte im hochpreisigen Luxussegment, indem sie auf die Entlegenheit und Unberührtheit australischer Farmlandschaften und die natürlichen Aufzucht- und Haltungsbedingungen ihrer Milchkühe verweisen. ${ }^{19}$ Die hier $\mathrm{zu}$ beobachtende Romantisierung und Inwertsetzung biophysisch-materieller Umweltfaktoren, die den landwirtschaftlichen Prozess prägen, ist sowohl aus der biologischen Landwirtschaft bekannt als auch aus der Debatte um geschützte geographische Herkunftsbezeichnungen landwirtschaftlicher Erzeugnisse. Kapitalistische Verwertungs- und Akkumulationsstrategien basieren hierbei nicht primär auf der Aneignung „Billiger Natur“ nach dem Verständnis Jason Moores, ${ }^{20}$ sondern vielmehr auf einer Kulturalisierung von Gütern ${ }^{21}$ - also der Aufladung agrarischer Güter mit einer symbolischen Bedeutung, die jedoch in den biophysisch-materiellen Umweltbedingungen des landwirtschaftlichen Produktionsprozesses wurzelt. Sind dies im Fall der Biolandwirtschaft meist ethische Werte, stehen bei chinesischen Konsumenten vor allem soziales Prestige und gesundheitliche Vorteile im Vordergrund, die mit dem Kauf hochpreisiger australischer Lebensmittel verbunden werden. Doch auch hier sehen sich Investoren durch die Materialität agrarischer Produktionsprozesse immer wieder herausgefordert. So zeigt Böhmes Fallstudie, dass eben diese angepriesene Abgeschiedenheit und die damit einhergehenden Transportschwierigkeiten ein wesentliches materielles Hindernis bei der Erwirtschaftung der anvisierten Renditen darstellen. ${ }^{22}$

19 M. Böhme, ,,Milk from the Purest Place on Earth‘. Examining Chinese Investments in the Australian Dairy Sector“, Agriculture and Human Values 38 (2021), S. 327-338.

20 J. Moore, „Über die Ursprünge unserer ökologischen Krise“, PROKLA 46 (2016) 185, S. 599 619.

21 Zum Begriff der „Kulturalisierung von Gütern“ siehe A. Reckwitz, Die Gesellschaft der Singularitäten. Zum Strukturwandel der Moderne, Berlin: Suhrkamp, 2018.

22 Böhme, ,Milk from the Purest Place on Earth'. 
Neuaushandlungen von Land, so lässt sich zusammenfassen, sind stets an materielle Aspekte von Land und Landwirtschaft gebunden. Auch wenn im Kontext des globalen Land Rush das Verhältnis zwischen Kapital und Land(-wirtschaft) zunehmend in einer neuen sozial-räumlichen Form - nämlich der der Finanzanlageklasse - gerinnt, bleibt die Analyse der Potentiale und Grenzen des Materiellen im kapitalistischen Akkumulationsprozess essentiell für das Verständnis der gegenwärtig zu beobachtenden Transformationsprozesse von Land. 